\title{
Harmonic generation in ablation plasmas of wide bandgap semiconductors
}

\author{
R. de Nalda, ${ }^{* a}$ M. López-Arias, ${ }^{a, b}$ M. Sanz, ${ }^{a}$ M. Oujja ${ }^{a}$ and M. Castillejo ${ }^{* a}$ \\ ${ }^{a}$ Instituto de Química Física Rocasolano, CSIC, Serrano 119, 28006 Madrid, Spain. \\ E-mail: r.nalda@iqfr.csic.es, m.castillejo@iqfr.csic.es \\ ${ }^{b}$ Unidad Asociada Departamento de Química Física I, Facultad de Ciencias Químicas, \\ Universidad Complutense de Madrid, 28040 Madrid, and Instituto de Estructura de la \\ Materia, CSIC, Serrano 123, 28006 Madrid, Spain
}

\begin{abstract}
Third and fifth harmonic generation of an IR $(1.064 \mu \mathrm{m})$ pulsed laser has been produced in ablation plasmas of the wide bandgap semiconductors CdS and ZnS. The study of the temporal behavior of the harmonic emission has revealed the presence of distinct compositional populations in these complex plasmas. Species ranging from atoms to nanometer-sized particles have been identified as emitters, and their nonlinear optical properties can be studied separately due to strongly differing temporal behavior. At short distances from the target $(<1 \mathrm{~mm})$, atomic species are mostly responsible for harmonic generation at early times ( $<500 \mathrm{~ns}$ ), while clusters and nanoaggregates mostly contribute at longer times $(>1 \mu \mathrm{s})$. Harmonic generation thus emerges as a powerful and universal technique for ablation plasma diagnosis and as a tool to determine the nonlinear optical susceptibility of ejected clusters or nanoparticles.
\end{abstract}




\section{Introduction}

Harmonic generation (HG) of intense laser pulses in gases has become a standard technique to create coherent pulses in the high-frequency region of the spectrum, from the UV to the XUV or X-ray. Whereas low-order harmonic generation has been possible for decades with laser pulses of nanosecond duration, and has constituted a common method for synthesizing VUV pulses for spectroscopy, ${ }^{1-3}$ high-order harmonic generation ${ }^{4}$ requires pulses of higher intensities (typically $>10^{15} \mathrm{~W} / \mathrm{cm}^{2}$ ) and has typically required the use of femtosecond driving lasers.

Harmonic generation experiments have been carried out mainly on atomic, ${ }^{5,6}$ or in some cases, molecular ${ }^{7,8}$ gas jets, but the phenomenon of HG is universal and can be generalized to other types of targets. In particular, it has been studied in ablation plasmas of a variety of materials. ${ }^{9-14}$ Laser ablation can easily generate exotic species (aggregates, polar molecules, highly ionized atoms) in the gas phase through the violent evaporation from a solid substrate and subsequent plasma dynamics, which involves aggregation, fragmentation and electron impact ionization. The nonlinear optical properties of such species, or their behavior under strong laser irradiation, can be studied through this technique. As examples, studies on clusters of rare gas atoms ${ }^{15-17}$ showed increased efficiencies of the harmonic yield that were attributed to collective electron oscillations. Less studies exist on harmonic generation in nanoparticles, ${ }^{12,14,18}$ but they have shown efficient harmonic generation, especially for low harmonic orders.

For compositionally simple ablation plasmas, the nonlinear behavior of a given species can be studied once it has been established that the desired species is the main harmonic emitter. ${ }^{9,11}$ For this purpose, it is important to provide an external diagnostic for the presence of the species, which can be correlated with the harmonic emission. This approach was successfully followed in ref. ${ }^{19}$, where harmonic emission was studied in the polar molecule $\mathrm{CaF}$ formed in the laser ablation of $\mathrm{CaF}_{2}$ crystals. In that study, the presence of CaF was independently monitored through laser-induced fluorescence (LIF). Alternatives to LIF include mass spectrometry-based techniques, or, as was realized in ref. ${ }^{13}$ for 
harmonic emission from nanoparticles, through the inspection of the ablation debris deposited on a nearby substrate.

In many cases, ablation plasmas are compositionally complex, and the study of the nonlinear behavior of a given species can only be made under certain conditions, i.e. either the species under study is the main or only harmonic emitter, or discrimination is possible through differing behavior of the components along time and/or space in the plasma. An alternative approach can be taken for the analysis of harmonic generation in complex plasmas: since virtually all species can emit harmonic radiation when subject to an intense laser pulse, HG can be conceived as a tool for plasma diagnosis, especially when performed in configurations that allow spatiotemporal exploration of different regions of the plasma. In this respect, albeit less specific, HG is highly versatile, and the limitations (mass range, spectral range, etc.) shown by other diagnostic techniques are absent.

Ablation plasmas of II-VI semiconductors constitute an excellent example of complex environments. Different techniques (mainly, optical emission spectroscopy and TOF-MS) have established the presence of neutral and ionized atoms, small molecules and a whole range of middle-sized aggregates. ${ }^{20-25}$ Larger aggregates with diameters of a few to some tens of nanometers are expected to be present in the plasma, since they have been seen in deposited form in pulsed laser deposition experiments. ${ }^{25,26}$ The only precursor work that shows results on HG on the plasma formed on the surface of semiconductors is ref. ${ }^{27}$, where Ganeev et al. studied high-order HG on InSb, InGaP, InP and GaAs plasmas, observing signatures of emission by molecular, rather than atomic species.

In this paper we report on low-order $\left(3^{\text {rd }}\right.$ and $\left.5^{\text {th }}\right)$ harmonic generation in species present in ablation plasmas of the semiconductors CdS and ZnS. As will be shown, temporal and spatial resolution across the plasma has allowed us to identify several distinct populations of harmonic emitters in such plasmas. We believe the interest of this work is twofold: on one hand, it shows the potential of the low harmonic generation technique for plasma diagnostics; on the other, it demonstrates that this technique can be used to study the nonlinear optical properties of complex species such as aggregates or nanoparticles. 


\section{Experimental}

The experimental setup was described in ref. ${ }^{19}$ and only a brief account will be given here. Ablation plasmas of $\mathrm{CdS}$ or $\mathrm{ZnS}$ were generated in vacuum through normal incidence irradiation with the second or fourth harmonic (532 or $266 \mathrm{~nm}$, respectively) of a Qswitched Nd:YAG laser (Quantel, Brilliant B, 6 ns FWHM) that was operated at $10 \mathrm{~Hz}$ using pulse energies of 1-10 mJ. This ablation beam was focused with a $17 \mathrm{~cm}$ focal length lens on the surface of the semiconductor target, generating maximum fluences of $\sim 5 \mathrm{~J} / \mathrm{cm}^{2}$, well above the ablation threshold. The vacuum chamber was kept at $5 \times 10^{-5} \mathrm{~Pa}$ background pressure, and the samples were mounted on a rotating holder to avoid cratering.

The harmonic driving laser was an IR pulse (Lotis TII LS-2147, 15 ns FWHM, 1064 nm) propagating parallel to the target surface in such a way that it intersected the ablation plasma at a controllable distance from the surface (distances between 0.6 and $2.1 \mathrm{~mm}$ were employed). Typical pulse energies were in the range of $50 \mathrm{~mJ}$ - $1 \mathrm{~J}$. The beam was focused with a $25 \mathrm{~cm}$ focal length lens, so that laser intensities in the harmonic generating region reached values of $\sim 10^{9} \mathrm{~W} / \mathrm{cm}^{2}$. The delay between this driving pulse and the ablation pulse was controlled electronically in the range from 0 to $50 \mu \mathrm{s}$.

Low-order harmonics of the driving laser were produced in the interaction region, and those propagated collinearly with the driving beam. Two highly IR transmissive, UV reflective mirrors were placed at the exit of the vacuum chamber to avoid damage of the detector with the intense IR beam. After the mirrors, a monochromator (TMc300 Bentham) with a ruled 1200 lines/mm grating was used for spectral discrimination. This system was coupled to a time-gated, intensified charge-coupled-device (ICCD, 2151 Andor Technologies) for detection of the generated harmonics. The third (at $355 \mathrm{~nm}$ ) and fifth (at $213 \mathrm{~nm}$ ) harmonics of the fundamental driving laser could be detected with this setup. Electronically excited species generated in the plasma produced optical emissions that could, under some conditions, be detected simultaneously with the harmonics. Typical acquisitions corresponded to an accumulation of 100 laser shots and a detection gate of 100 ns. 
The CdS and ZnS targets were prepared from CdS and ZnS powders (Alfa Aesar 99.999\%, particle sizes $<44 \mu \mathrm{m}$ and $<10 \mu \mathrm{m}$, respectively) that were pelletized into disks of $10 \mathrm{~mm}$ diameter and about $2 \mathrm{~mm}$ of thickness using a hydrostatic press at $8 \mathrm{ton} / \mathrm{cm}^{2}$ followed by sintering at $350{ }^{\circ} \mathrm{C}$ in air for $12 \mathrm{~h}$.

\section{Results and discussion}

Spectra were acquired in the region of interest for a given distance of the driving laser from the sample surface and for a range of temporal delays. In those measurements, spontaneous emissions from excited species in the plume were recorded simultaneously with the third harmonic (TH) of the IR driving laser. An example of such data can be seen in Figure 1, where detected spectra in the range of 320 to $370 \mathrm{~nm}$ from a 266-nm plasma of a CdS sample, with the IR driving laser propagating at a distance of $1 \mathrm{~mm}$ from the surface, are presented. The three panels correspond to different delay times with respect to the ablation event. The third harmonic of the driving field, at $355 \mathrm{~nm}$, can be observed at all delays in the range, with maximum intensity around $250 \mathrm{~ns}$ (panel (b) in the Figure). Simultaneously, we detect the presence of electronically excited neutral $\mathrm{Cd}$ through its spontaneous emissions marked (1)-(4) in the Figure. Assignments are shown in the Figure caption. Those emissions appear Stark broadened for short delays due to the high electron density. It is important to realize that the detection of emissions corresponds spatially to the overall plume, since no imaging is performed. Additional emissions corresponding to neutral and singly ionized Cd could be detected in other spectral ranges.

The fifth harmonic at $213 \mathrm{~nm}$ could be detected either in its second diffraction order with the same system, or in first order with a PMT detector.

As a further confirmation of the nature of the emission detected at $355 \mathrm{~nm}$, studies of the power dependence with respect to the energy of the driving beam were performed for the two materials. Figure 2 shows examples of those measurements for $266 \mathrm{~nm}$ generated plasmas of CdS and ZnS. The slopes obtained in log-log plots were compatible with the value of 3 , expected for the behavior of the third harmonic in the perturbative regime. 
Slopes obtained for the fifth harmonic (not shown) were compatible with the expected value of 5 .

In the rest of the paper we will explore the behavior of the emitted harmonics (we will concentrate on the $\mathrm{TH}$ ) as a function of the temporal-spatial region of the plume that is sampled by the driving laser. In each case, the ablation laser energy is indicated. Changes in this value produce quantitative changes in the central positions of the different components and in some cases, on their relative intensity, but the general features remain unchanged.

Figure 3(a) shows the emitted TH in a UV-produced CdS plume, at two distances from the surface of the sample $(0.6 \mathrm{~mm}$ and $1.0 \mathrm{~mm})$ and as a function of the time delay between the ablation event and the arrival of the IR driving laser. The curves obtained have been normalized for easier comparison. Two temporal components are observed at both distances, the strongest one corresponding to short delays (peaking at $150 \mathrm{~ns}$ for $0.6 \mathrm{~mm}$ distance and at $250 \mathrm{~ns}$ for $1.0 \mathrm{~mm}$ distance) and a weaker one (by more than one order of magnitude) that temporally extends to around $2 \mu$ s (at $0.6 \mathrm{~mm}$ ) or $3 \mu$ s (at $1.0 \mathrm{~mm}$ ). This type of behavior seems to be indicative of the presence of two components of the plasma that can generate $\mathrm{TH}$, either compositionally or in terms of kinetic energy. For further investigation, a set of z-scan measurements were performed, i.e. measurements of the TH as a function of the position of the focus of the driving laser with respect to the center of the plasma, in the direction of propagation. Under all conditions tested, a single maximum, corresponding to focusing the driving laser at the center of the plasma, was found. The results obtained through z-scans for all conditions are shown in Table 1. For 266-nm plasmas of CdS, the $z$-scans were performed under the conditions marked with arrows on Figure 3(a), and the results are shown in Figures 3(b), 3(c) and 3(d). The data were fitted with gaussian functions. Large values were obtained for the full-width-half-maximum (FWHM) of those measured in the temporal region of maximum TH $(11 \mathrm{~mm}$ for $0.6 \mathrm{~mm}$ distance, at $150 \mathrm{~ns}$, Fig. 3(b), and $13 \mathrm{~mm}$ for $1 \mathrm{~mm}$ distance, at $250 \mathrm{~ns}$, Fig. 3(c)). In the case of the second component, a much narrower structure is found along the propagation direction, with a FWHM of $5.6 \mathrm{~mm}$ at $0.6 \mathrm{~mm}$ distance, when the measurement is acquired 
at a delay of $800 \mathrm{~ns}$. This indicates that the second, slower component, is emitted much more directionally than the stronger, faster one.

The data shown in Figure 3 indicate that the first temporal component is spatially broad over the plane perpendicular to the normal to the surface, i.e. it is emitted over an angularly broad distribution. Indeed, a measurement of the intensity of this signal as a function of the distance to the target $r$, for the delay that corresponds to maximum $\mathrm{TH}$, yields a fit compatible with HG $\alpha r^{-4}$. This is the expected behaviour for an isotropic particle emission (density $\alpha r^{-2}$ ), given that for an optical nonlinear process, we expect HG to be proportional to the square of the density. The behavior of the second component as a function of distance was not measurable due to the poorer signal-to-noise ratio.

The case of the ZnS ablation plasma at $266 \mathrm{~nm}$ presents similarities but also some differences with respect to CdS. Figure 4 shows the corresponding measurements for the TH taken at the same two distances (0.6 and $1.0 \mathrm{~mm}$ from the sample) as a function of delay. The presence of two components, or populations, is still clear at $0.6 \mathrm{~mm}$, but the distinction is lost at $1.0 \mathrm{~mm}$. Z-scan measurements yield the results shown in Table 1 . The data show that the two populations are still distinguishable through their TH emission at 0.6 $\mathrm{mm}$, and possess different $z$-scan behavior, but this is no longer the case at $1.0 \mathrm{~mm}$. At this longer distance, the fact that both at short and long delay the distribution presents similar width along the $z$ direction indicates that it can be considered that a single population is being sampled.

Hence, for 266-nm ablation, at short distance from the target ( $\leq 1 \mathrm{~mm})$, both in CdS and $\mathrm{ZnS}$, an early, temporally sharp component is identified in the region $100-200$ ns. This is followed by a much broader component (temporally) that can be observed at temporal delays up to $\sim 3 \mu \mathrm{s}$. As the distance from the target is increased, both components are temporally delayed, as expected, and the early, temporally sharp component becomes significantly less intense with respect to the slower, temporally broader component. For ZnS this early component is no longer visible for $d>0.6 \mathrm{~mm}$; for CdS, it disappears for $d>$ $2 \mathrm{~mm}$. In view of the results of the $z$-scans, this can be attributed to the very different 
angular nature of the two components (very broad, almost isotropic, for the early component, and much more directional for the slow component).

Upon $532 \mathrm{~nm}$ ablation, the spatiotemporal behavior of HG presents some interesting differences with respect to UV ablation. In CdS, the same two populations appear, peaking around $200 \mathrm{~ns}$ and $1000 \mathrm{~ns}$ for a distance of $1.0 \mathrm{~mm}$, and they behave similarly to $266 \mathrm{~nm}$ with respect to the distance from the target, i.e. longer delays are found for larger distance, and larger slow-to-fast signal ratios are found for larger distance. The data are shown in Figure 5. Note that at the shortest distance used, of $0.6 \mathrm{~mm}$, the two populations are undistinguishable. However, the slow-to-fast signal ratio is considerably more favourable at 532 than at $266 \mathrm{~nm}$, revealing plumes that are richer in middle-size aggregates (see discussion below). Note that the y-axis of Figure 5 is now in linear scale, since this now allows a better comparison of the two components. As was the finding for $266 \mathrm{~nm}$, the fast component is angularly broad, causing a fast disappearance of the signal as the distance from the surface is increased, and the slow component is much more directional.

An additional probe of the contents of the plume was found for $532 \mathrm{~nm}$ ablation of CdS in the form of two induced emissions observed at 514 and $548 \mathrm{~nm}$. They were assigned to second diffraction orders of $\mathrm{Cd}^{+}$emissions at 257.3 and $274.8 \mathrm{~nm}^{28}$ The observation of these emissions was coincident with the visual observation of an intense white luminescence from the region of the plume irradiated with the IR driving laser. The temporal behavior of these emissions was studied, and the dependences were found to be very similar to the slow component of THG. We tentatively assign these emissions to the production of electronically excited $\mathrm{Cd}^{+}$as a result of IR-induced fragmentation processes in CdS aggregates.

The TH behaviour in 532-nm ablation plasmas of $\mathrm{ZnS}$ is of particular interest. Figure 6 shows the behavior of the TH at a fixed distance of $1.0 \mathrm{~mm}$ and for three different ablation laser energies. As had been found at $266 \mathrm{~nm}$ ablation, two populations appear centered around 200 and 1000 ns. For moderate fluence (laser pulse energy $<2 \mathrm{~mJ}$ ), the first component is relatively weak, with the slower species dominating $\mathrm{TH}$ generation. In this 
case, the investigation of three different ablation energies has shown that the intensity ratio of the fast vs. slow component is higher for higher intensity. The interesting feature in these conditions is the fact that an additional temporal component was found for very long delay times $(\sim 10 \mu \mathrm{s})$ with respect to the ablation event. This component extends temporally to delays up to $\sim 50 \mu \mathrm{s}$.

We will next discuss the identity of the two (or three for 532-nm ZnS plasmas) components probed by HG in these semiconductor plasmas. In all conditions, a first component is observed that is temporally centered around 100-300 ns for distances from the target of $\sim 1$ mm. It is clear that this component should be assigned to the presence of atomic species in the plasma, i.e. Cd, Zn and S, with velocities around $5 \times 10^{5} \mathrm{~cm} / \mathrm{s}$. This type of velocity is typical under these conditions (see for instance ref. ${ }^{29}$ for Cd I velocities upon CdS ns laser ablation, or ref. ${ }^{30}$ for $\mathrm{Zn}$ I velocities in a $\mathrm{ZnO}$ ablation plume). Frequency tripling in $\mathrm{Cd}$ vapor was reported as early as $1972^{31}$ and $\mathrm{Cd}$ has been used as an efficient nonlinear medium for VUV generation in later works. ${ }^{32,33}$. With respect to the angular behavior, some studies on angular distribution of particles emitted in a nitrogen laser desorption process in CdS have been reported ${ }^{34}$. Namiki et al. report the behavior of the desorption flux and find strong dependences of the kinetic energy content of the atomic species as a function of the polar angle. However, in their low fluence work, the speeds are much lower than those found in our conditions, so that direct comparison is not possible. The findings of the present work show that this fast component, mainly consisting of atomic species, present a broad angular character.

The second component has been found to be typically centered at $\sim 1 \mu$ s for the same distance, i.e. corresponding to velocities around $1 \times 10^{5} \mathrm{~cm} / \mathrm{s}$. We believe this component should be assigned to clusters or aggregates formed in the ablation plasma. In TOF-MS studies on ns ablation plumes of these semiconductors, a broad range of middle-sized aggregates $(\mathrm{CdS})_{n}$ or $(\mathrm{ZnS})_{n}$, with $n$ in the range from 2 to 30, have been found by us and other authors. $^{20-24}$ These aggregates constitute a much larger fraction of the observed species for ablation with wavelengths in the visible than in the UV. This is in agreement 
with the findings of the present work, where the second component, relative to the first, is considerably more prominent for 532-nm plasmas than for 266-nm plasmas.

As was shown in Figure 6, a third component was observed for ZnS plasmas generated in a $532 \mathrm{~nm}$ ablation process, temporally centered around $10 \mu$ s and extending to long delay times as long as $50 \mu \mathrm{s}$. The estimated central velocity for this component is $10^{4} \mathrm{~cm} / \mathrm{s}$. We believe that this component can be constituted by nanoparticles with diameters of a few nanometers. This type of particles has been found both through their broadband emission in ultrashort laser ablation of a wide range of materials: $\mathrm{Au}$ and $\mathrm{Ag}$ (noble metals), $\mathrm{Ni}$ (ferromagnetic metal), $\mathrm{Si}$ (semiconductor), or $\mathrm{TbDyFe}$ and $\mathrm{MgB}_{2}$ (superconducting multicomponents), as has been shown for instance in refs. ${ }^{35}$ and ${ }^{36}$. A common characteristic of the optical emission spectra reported at $1 \mathrm{~mm}$ distance from the target surface for all those materials was a slow component, appearing typically between 5 and $100 \mu$ s after ablation (i.e. with velocities compatible with those found in the present work). In those cases, deposits showed the presence of nanoparticles of the order of $10 \mathrm{~nm}$ diameter on the substrate. Broadband emission from hot nanoparticles is not as universal for nanosecond as it is for femtosecond laser ablation, but it has nevertheless been described in a variety of materials. ${ }^{37,38}$ This work provides a confirmation of their presence in visible laser induced ablation plasmas of $\mathrm{ZnS}$.

The assumption of a totally statistical ablation process supports the above assignments. In effect, if all components are assumed to reach thermodynamical equilibrium in the first stages, so that the same average kinetic energy is expected for all compositional families of the plasma, we could estimate average sizes of the second and third components once the first component is assigned to the atomic species. For $\mathrm{ZnS}$, if the first component $\left(5 \times 10^{5}\right.$ $\mathrm{cm} / \mathrm{s})$ is assumed to be mainly due to $\mathrm{Zn}$ atoms, the second population $\left(1 \times 10^{5} \mathrm{~cm} / \mathrm{s}\right)$ should be centered at $(\mathrm{ZnS})_{10}$, with a range that is approximately between $(\mathrm{ZnS})_{3}$ and $(\mathrm{ZnS})_{40}$. This corresponds to the range of clustered species that have been shown to appear in TOF-MS experiments. ${ }^{20-24}$ Under this assumption, the third population $\left(10^{4} \mathrm{~cm} / \mathrm{s}\right)$ should be centered at $(\mathrm{ZnS})_{500}$. Using an average $\mathrm{ZnS}$ lattice constant of $5 \AA$, these particles would be expected 
to have a diameter of $6 \mathrm{~nm}$. This is consistent with typical diameters found for particles synthesized through PLD. ${ }^{25,26,35,39,40}$

The observations are also consistent with trends found in TOF-MS experiments by some of the authors, ${ }^{23,24}$ where atomic and middle-sized aggregates can be detected. In those experiments, performed for CdS targets, clear differences could be observed in the composition of the plumes for UV vs. visible irradiation (much higher proportion of clusters for visible lasers) and for low vs. high fluences (higher proportion of clusters for low fluence) that are consistent with the findings of this work.

The observation of multicomponent ablation plumes has been reported in numerous occasions in the literature (refs. ${ }^{35,41,42}$ and references therein) and the most standard technique for their detection has been optical emission spectroscopy (OES). The main drawback of OES is that it requires relatively high temperatures for detection to be possible in the accessible visible range. In fact, temperatures of the nanoparticles that are too low for their blackbody radiation to enter into the visible range has been indicated as a possible reason for their apparent absence in some cases. ${ }^{25}$ In contrast to this limitation, HG is universal with respect to composition, size, or temperature of the sample.

The technique described in this work could be used in certain cases for the measurement of the optical nonlinear susceptibility of clusters or nanoparticles. For the species under study here, Cheng et al. ${ }^{43}$ reported a decrease in third-order susceptibility of CdS clusters with decreasing size, using clusters of 1.5 and $3.0 \mathrm{~nm}$ diameter. However, the solvents and mole fractions were dramatically different, so that comparison was difficult. Nosaka et al. ${ }^{44}$ performed a study of TH generation of CdS nanoparticles embedded in PVC. In a frequency conversion scheme identical to that employed in the present work (1064 nm to $355 \mathrm{~nm}$ ), they observed that the optical nonlinearity remained practically constant in the diameter range studied (2-4 $\mathrm{nm})$.

The enhanced nonlinear response of nanoparticle-sized species is related to their quantum confinement. High values of the third-order nonlinear susceptibility can be found, 
especially near the surface plasmon resonances. Enhancement of the third harmonic generation in clusters of noble gases was explored in ref. ${ }^{16}$ and later in ${ }^{45}$, where it was found that optimum TH was originated from a relatively narrow range of cluster sizes, where both the nonlinear optical response and the coherence length of the cluster medium reached maximum values. Given that ablation plasmas, especially in carefully controlled irradiation and expansion conditions, can constitute good cluster sources, the technique described in the present work, where TH is explored with temporal and spatial resolution, can provide a powerful tool to study the nonlinear optical response of these forms of matter.

\section{Conclusions}

The study of low-order harmonic generation in ablation plasmas of the wide bandgap semiconductors CdS and ZnS has revealed a rich spatiotemporal structure, where harmonics are emitted from species ranging from atoms and small molecules to nanometersized aggregates. Discrimination is made possible through the use of adequate geometries and a two-laser pulse sequence that permits the exploration of the compositionally distinct regions of the plasma. Through the use of this technique, we have observed profound differences in the composition of these plasmas as a function of the ablation laser wavelength. In particular, for photon energies above the material bandgap, low-mass species (mainly atomic) dominate, whereas photon energies below the bandgap strongly favor the formation of more massive species, both clusters and nanoparticles. The fact that low-order harmonic generation is universal makes it an ideal tool for the in situ study of complex media like ablation plasmas. The measurement of the nonlinear susceptibilities of exotic species (clusters, nanoaggregates) should be made possible especially when it can be applied in parallel with an independent technique like optical emission spectroscopy. Extension to ultrashort laser-induced plasmas, which are rich in aggregates created temporally near the ablation event, would provide a powerful method for the study of nonlinear optical properties of these species in environment-free conditions. 


\section{Acknowledgements}

Funding has been provided by Ministry of Science and Innovation of Spain (MICINN) under Project CTQ2007-60177. We thank support from EU ITN network FASTQUAST (PITN-GA-2008-214962). M.S. and M.O. acknowledge CAM (Geomateriales P2009/MAT 1629) and CONSOLIDER CSD2007-00058 respectively for contracts. M. L.-A. thanks CSIC for a JAE-Pre contract. 


\section{References}

1 R. Hilbig and R. Wallenstein, IEEE J. Quantum Elec., 1981, 17, 1566.

2 A.H. Zanganeh, J. H. Fillion, J. Ruiz, M. Castillejo, J. L. Lemaire, N. Shafizadeh and F. Rostas, J. Chem. Phys., 2000, 112, 5660.

3 C.Y. Ng, Ann. Rev. Phys. Chem., 2002, 53, 101.

4 J.J. Macklin, J.D. Kmetec and C.L. Gordon, Phys. Rev. Lett., 1993, 70, 766.

5 X.F. Li, A. L'Huillier, M. Ferray, L.A. Lompre and G. Mainfray, Phys. Rev. A, 1989, 39, 5751 .

6 C. Spielmann, N.H. Burnett, S. Sartania, R. Koppitsch, M. Schnurer, C. Kan, M. Lenzner, P. Wobrauschek and F. Krausz, Science, 1997, 278, 661.

7 N. Hay, R. de Nalda, T. Halfmann, K.J. Mendham, M.B. Mason, M. Castillejo and J.P. Marangos, Eur. Phys. J. D, 2001, 14, 231.

8 J. Levesque, Y. Mairesse, N. Dudovich, H. Pepin, J.C. Kieffer, P.B. Corkum and D.M. Villeneuve, Phys. Rev. Lett., 2007, 99, 243001.

9 W. Theobald, C. Wülker, F.P. Schäfer and B.N. Chichkov, Opt. Comm., 1995, 120, 177.

10 R.A. Ganeev, J. Phys. B: At. Mol. Opt. Phys., 2007, 40, R213.

11 R. A. Ganeev, L. B. Elouga Bom, J. Abdul-Hadi, M. C. H. Wong, J. P. Brichta, V. R. Bhardwaj and T. Ozaki, Phys. Rev. Lett., 2009, 102, 013903. 
12 R.A. Ganeev, M. Suzuki, M. Baba and H. Kuroda, Appl. Phys. Lett., 2009, 94, 051101.

13 R.A. Ganeev, L.B. Elouga Bom and T. Ozaki, J. Appl. Phys., 2009, 106, 023104.

14 H. Singhal, R.A. Ganeev, P.A. Naik, J.A. Chakera, U. Chakravarty, H.S. Vora, A.K. Srivastava, C. Mukherjee, C.P. Navathe, S.K. Deb and P.D. Gupta, Phys. Rev. A, 2010, 82, 043821.

15 T.D. Donnelly, T. Ditmire, K. Neuman, M.D. Perry and R.W. Falcone, Phys. Rev. Lett., 1996, 76, 2472.

16 C. Vozzi, M. Nisoli, J-P. Caumes, G. Sansone, S. Stagira, S. De Silvestri, M. Vecchiocattivi, D. Bassi, M. Pascolini, L. Poletto, P. Villoresi and G. Tondello, Appl. Phys. Lett., 2005, 86, 111121.

17 J.W.G. Tisch, T. Ditmire, D.J. Frasery, N. Hay, M.B. Mason, E. Springate, J.P. Marangos and M.H.R. Hutchinson, J. Phys. B, 1997, 30, L709.

18 R. A. Ganeev, M. Suzuki, M. Baba, M. Ichihara and H. Kuroda, J. Appl. Phys., 2008, 103, 063102.

19 M. Oujja, R. de Nalda, M. López-Arias, R. Torres, J. P. Marangos and M. Castillejo, Phys. Rev. A, 2010, 81, 043841.

20 T.P. Martin, Shells of atoms, Phys. Rep., 1996, 273, 199.

21 A.Burnin and J.J. BelBruno, Chem. Phys. Lett., 2002, 362, 341.

22 E. Sanville, A. Burnin and J.J. BelBruno, J. Phys. Chem. A, 2006, 110, 2378.

23 J. Alvarez, M. Lopez-Arias, R. de Nalda and M. Martín, Appl. Phys. A, 2008, 92, 831. 
24 J. Álvarez-Ruiz, M. López-Arias, R. de Nalda, M. Martín, A. Arregui and L. Bañares, Appl Phys. A, 2009, 95, 681.

25 M. Sanz, M. López-Arias, J. F. Marco, R. de Nalda, S. Amoruso, G. Ausanio, S. Lettieri, R. Bruzzese, X. Wang and M. Castillejo, submitted (2010).

26 M. Sanz, R. de Nalda, J. F. Marco, J. G. Izquierdo, L. Bañares and M. Castillejo, J. Phys. Chem. C, 2010, 114, 4864.

27 R.A. Ganeev and P.V. Redkin, Opt. Comm., 2008, 281, 4126.

28 NIST Atomic Spectra Database, http://physics.nist.org.

29 H. Ezumi and S. Keitoku, Jpn. J. Appl. Phys., 1993, 32, 1783.

30 A.Klini, A. Manousaki, D. Anglos and C. Fotakis, J. Appl. Phys., 2005, 98, 123301.

31 A.H. Kung, J.F. Young, G.C. Bjorklund and S.E. Harris, Phys. Rev. Lett., 1972, 29, 985.

32 K. Miyazaki, H. Sakai and T. Sato, Opt. Lett., 1984, 9, 457.

33 H. Sakai and K. Miyazaki, Jpn. J. Appl. Phys., 1992, 31, 3354.

34 A.Namiki, T. Kawai and K. Ichige, Surface Science, 1986, 166, 129.

35 S. Amoruso, R. Bruzzese, N. Spinelli, R. Velotta, M. Vitiello and X. Wang, Europhys. Lett., 2004, 67, 404.

36 S. Amoruso, G. Ausanio, R. Bruzzese, M. Vitiello and X. Wang, Phys. Rev. B, 2005, 71, 033406. 
37 E.A. Rohlfing, J. Chem. Phys., 1988, 89, 6103.

38 K. Sasaki, T. Wakasaki and K. Kadota, Appl. Surf. Sci., 2002, 197, 197.

39 M. Sanz, M. Walczak, M. Oujja, A. Cuesta and M. Castillejo, Thin Solid Films, 2009, $517,6546$.

40 M. Sanz, M. Walczak, R. de Nalda, M. Oujja, J.F. Marco, J. Rodríguez, J.G. Izquierdo, L. Bañares and M. Castillejo, Appl. Surf. Sci., 2009, 255, 5206.

41 O. Eryu, K. Murakami, K. Masuda, A. Kasuya and Y. Nishina, Appl. Phys. Lett., 1989, 54, 2716.

42 J. Perrière, C. Boulmer-Leborgne, R. Benzerga and S. Tricot, J. Phys. D: Appl. Phys., 2007, 40, 7069.

43 L.-T. Cheng, N. Herron and Y. Wang, J. Appl. Phys., 1989, 66, 3417.

44 Y. Nosaka, K. Tanaka and N. Fujii, Journal of Applied Polymer Science, 1993, 47, 1773.

45 B. Shim, G. Hays, R. Zgadzaj, T. Ditmire and M. C. Downer, Phys. Rev. Lett., 2007, 98, 123902.

46 K. Burns and K.B. Adams, J. Opt. Soc. Am., 1956, 46, 94. 
Table 1. Full-width-half maximum of THG emission as a function of position of IR driving laser focus with respect to center of ablation plasma in the direction of laser propagation (zscan).

\begin{tabular}{|l|l|l|l|l|}
\hline & $0.6 \mathrm{~mm}, 150 \mathrm{~ns}$ & $0.6 \mathrm{~mm}, 800 \mathrm{~ns}$ & $1.0 \mathrm{~mm}, 250 \mathrm{~ns}$ & $1.0 \mathrm{~mm}, 900 \mathrm{~ns}$ \\
\hline CdS, 266 nm & 11 & 5.6 & 13 & ----------------- \\
\hline ZnS, 266 nm & 8.6 & 5.8 & 5.4 & 6.7 \\
\hline CdS, $532 \mathrm{~nm}$ & ----------------- & 9.2 & ---------------- & 6.0 \\
\hline
\end{tabular}




\section{Figure captions}

Figure 1. Optical spontaneous emissions ((1)-(4)) in the spectral range 320-370 nm from a CdS plume generated through 266-nm, $5 \mathrm{~mJ}$ laser irradiation, together with the third harmonic (TH, $355 \mathrm{~nm}$ ) of a $1064 \mathrm{~nm}$ driving pulse propagating across the plume at $1 \mathrm{~mm}$ from the surface. The emissions can be assigned to neutral Cd in the transitions (1) $7 \mathrm{~s}{ }^{3} \mathrm{~S}_{1}$ $\rightarrow 5 \mathrm{p}{ }^{3} \mathrm{P}_{2}, 5 \mathrm{p}{ }^{3} \mathrm{P}_{1} \rightarrow 5 \mathrm{~s}^{2}{ }^{1} \mathrm{~S}_{0}$ (2) $5 \mathrm{~d}{ }^{3} \mathrm{D}_{1} \rightarrow 5 \mathrm{p}{ }^{3} \mathrm{P}_{0}$ (3) $5 \mathrm{~d}{ }^{3} \mathrm{D}_{1,2} \rightarrow 5 \mathrm{p}{ }^{3} \mathrm{P}_{1}$ (4) $5 \mathrm{~d}{ }^{3} \mathrm{D}_{1,2,3} \rightarrow 5 \mathrm{p}$ ${ }^{3} \mathrm{P}_{2}$ (ref. ${ }^{46}$ ). Panels (a), (b) and (c) correspond to delays of 50, 250 and 1000 ns, respectively, between the ablation event and the arrival of the driving laser pulse. Signal intensities are in arbitrary units, but relative intensities in the three panels correspond to measured values.

Figure 2. Log-log plot of the intensity of the third harmonic (355 $\mathrm{nm}$ ) of the driving laser as function of the laser intensity, for $266 \mathrm{~nm}$ ablation of a CdS sample (open circles) and a ZnS sample (solid circles). The slope is compatible with a cubic dependence in both cases.

Figure 3. (a) Third harmonic of the IR driving laser in a CdS ablation plasma induced by a $266 \mathrm{~nm}$ ablation laser (3.6 mJ), as a funcion of the delay between the ablation event and the arrival of the driving laser, and for two different distances from the surface of the target. The TH intensity is plotted in log scale for better inspection. (b), (c) and (d) z-scans where the TH is measured as a function of the position of the IR focus $(z)$ with respect to the center of the ablation plasma in the direction of propagation. The conditions were the following: (b) distance to target: $0.6 \mathrm{~mm}$, delay = $150 \mathrm{~ns}$; (c) distance to target: $1.0 \mathrm{~mm}$, delay = $250 \mathrm{~ns}$; (d) distance to target: $0.6 \mathrm{~mm}$, delay $=800 \mathrm{~ns}$.

Figure 4. Third harmonic of the driving laser in a ZnS ablation plasma induced by a 266 $\mathrm{nm}$ ablation laser $(3.7 \mathrm{~mJ})$, as a funcion of the delay between the ablation event and the propagation of the driving laser, and for two different distances from the surface. The TH intensity is plotted in log scale for better inspection. 
Figure 5. Third harmonic of the driving laser in a CdS ablation plasma induced by a 532 $\mathrm{nm}$ ablation laser $(2.4 \mathrm{~mJ})$, as a funcion of the delay between the ablation event and the propagation of the driving laser, and for three different distances from the surface. It is important to note that, as opposed to Figures 3 and 4, in this case the y-axis is in linear scale.

Figure 6. Third harmonic of the IR driving laser in a ZnS ablation plasma induced by a 532 $\mathrm{nm}$ ablation laser, as a funcion of the delay between the ablation event and the propagation of the driving laser, at $1.0 \mathrm{~mm}$ from the surface. The graphs are plotted in log-log scale for better inspection. The three panels correspond to different ablating laser pulse energies: (a) $\mathrm{E}_{\text {ablation }}=0.8 \mathrm{~mJ}$, (b) $\mathrm{E}_{\text {ablation }}=1.5 \mathrm{~mJ}$, (c) $\mathrm{E}_{\text {ablation }}=2.5 \mathrm{~mJ}$. Dashed lines have been drawn to indicate the central temporal positions of the three populations found. 


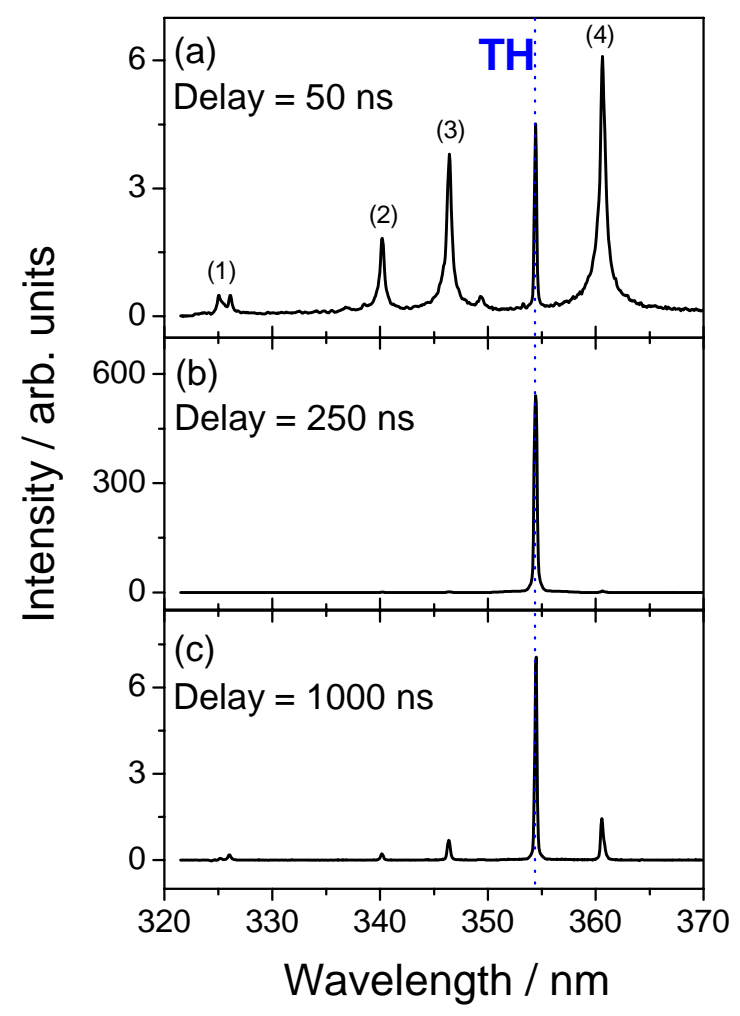

Figure 1 


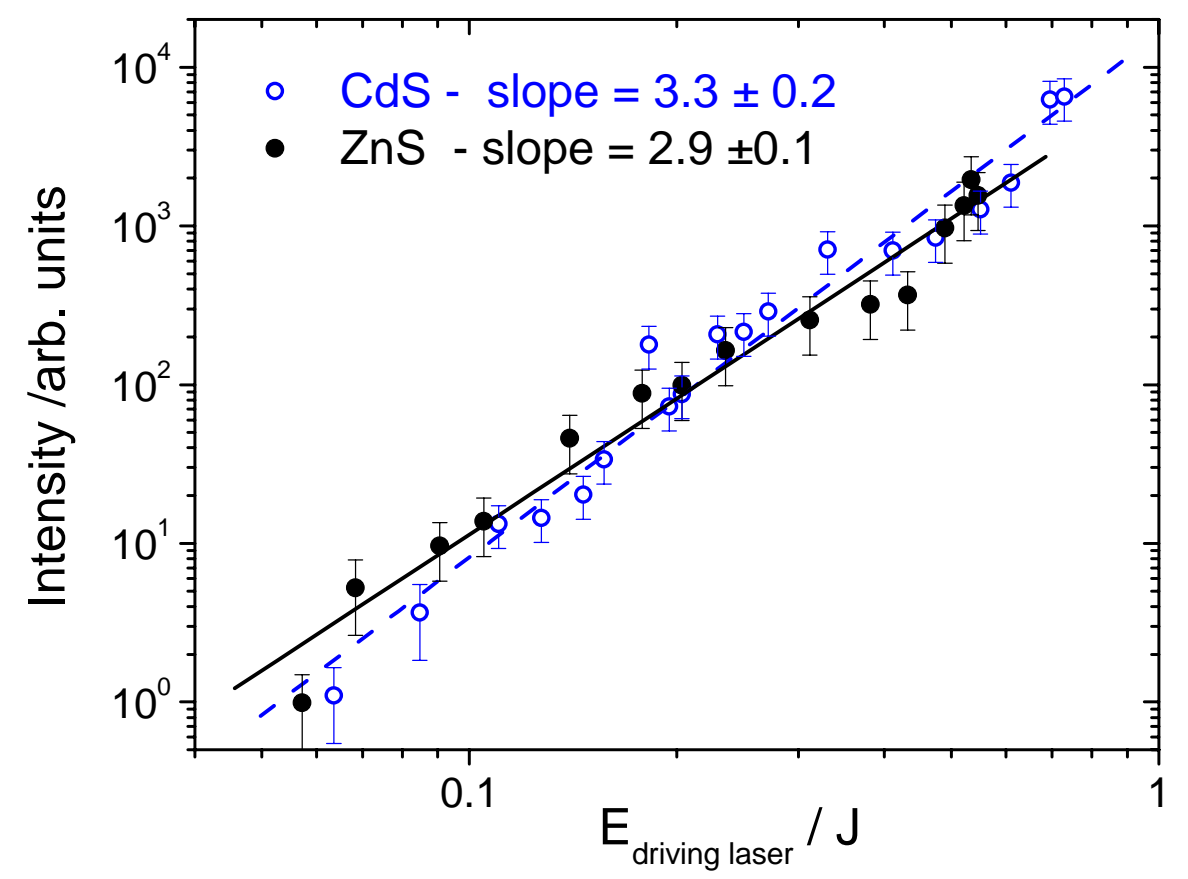

Figure 2 

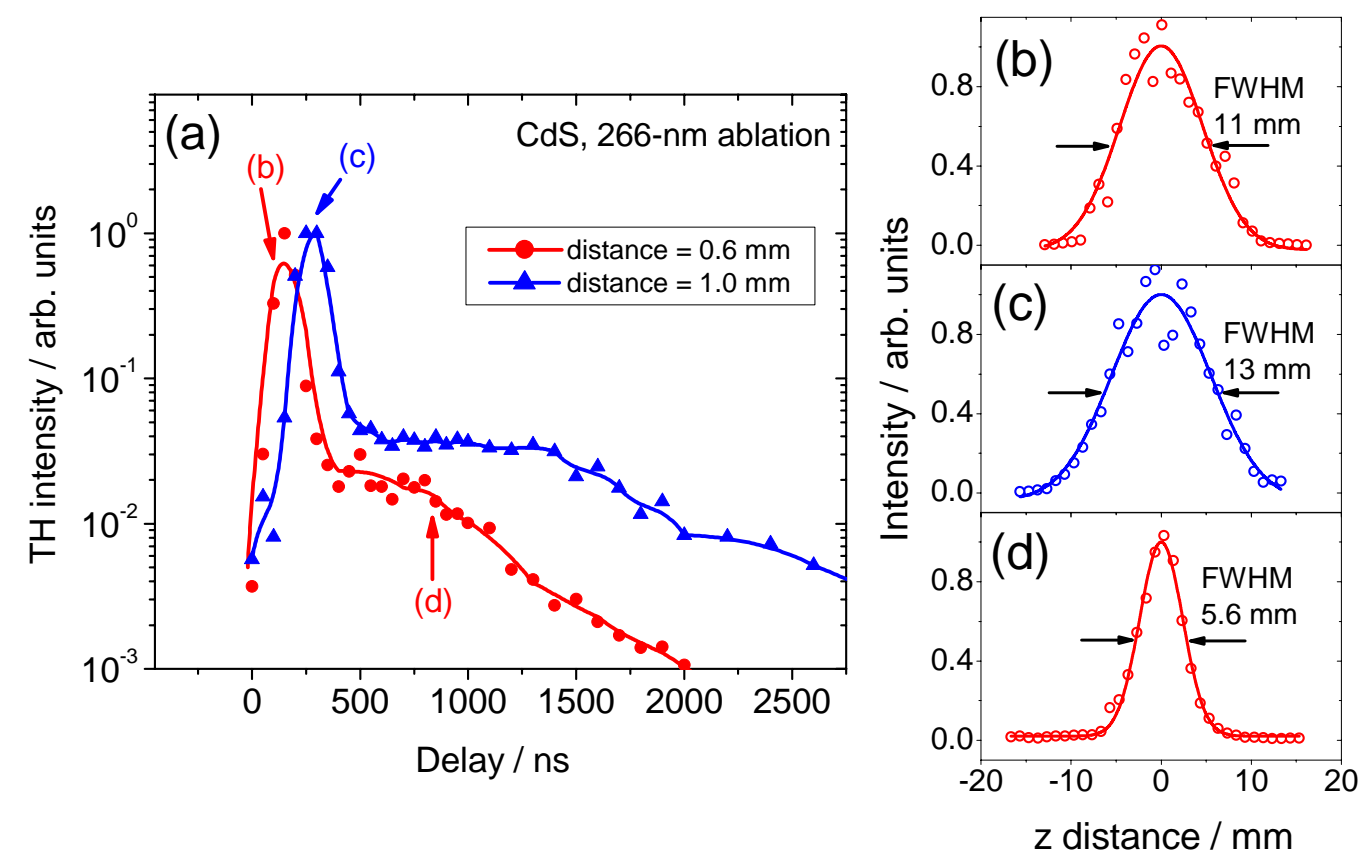

Figure 3 


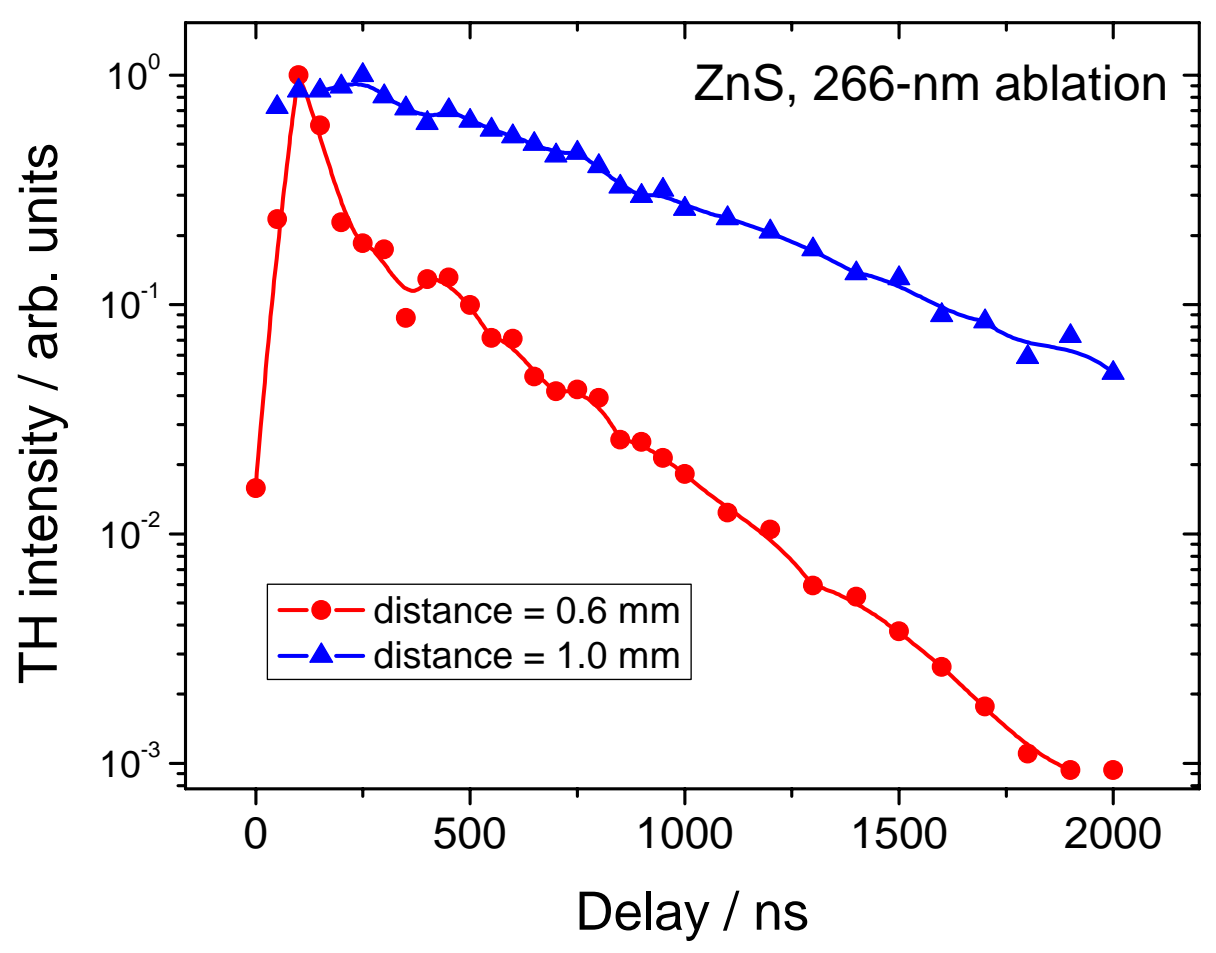

Figure 4 


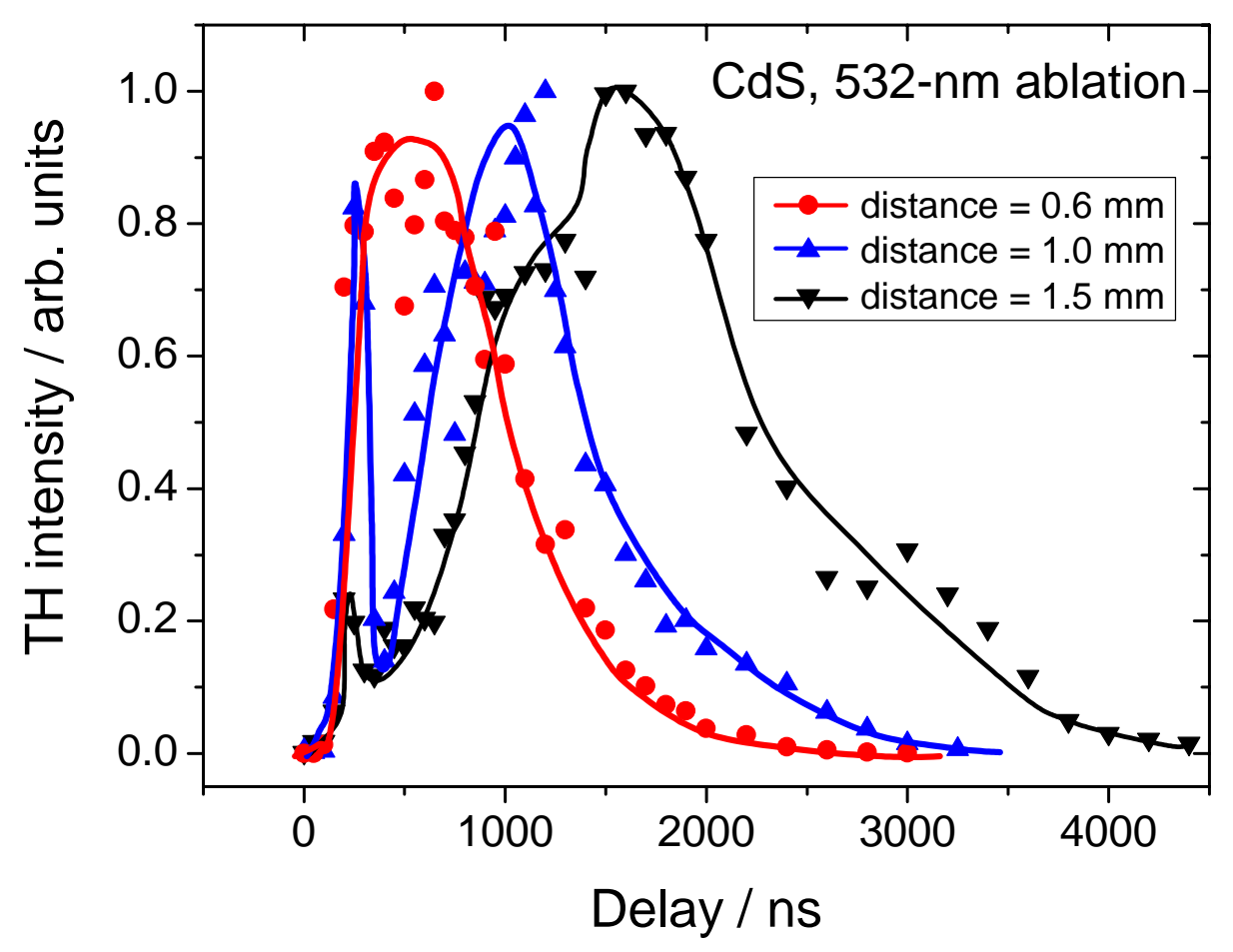

Figure 5 


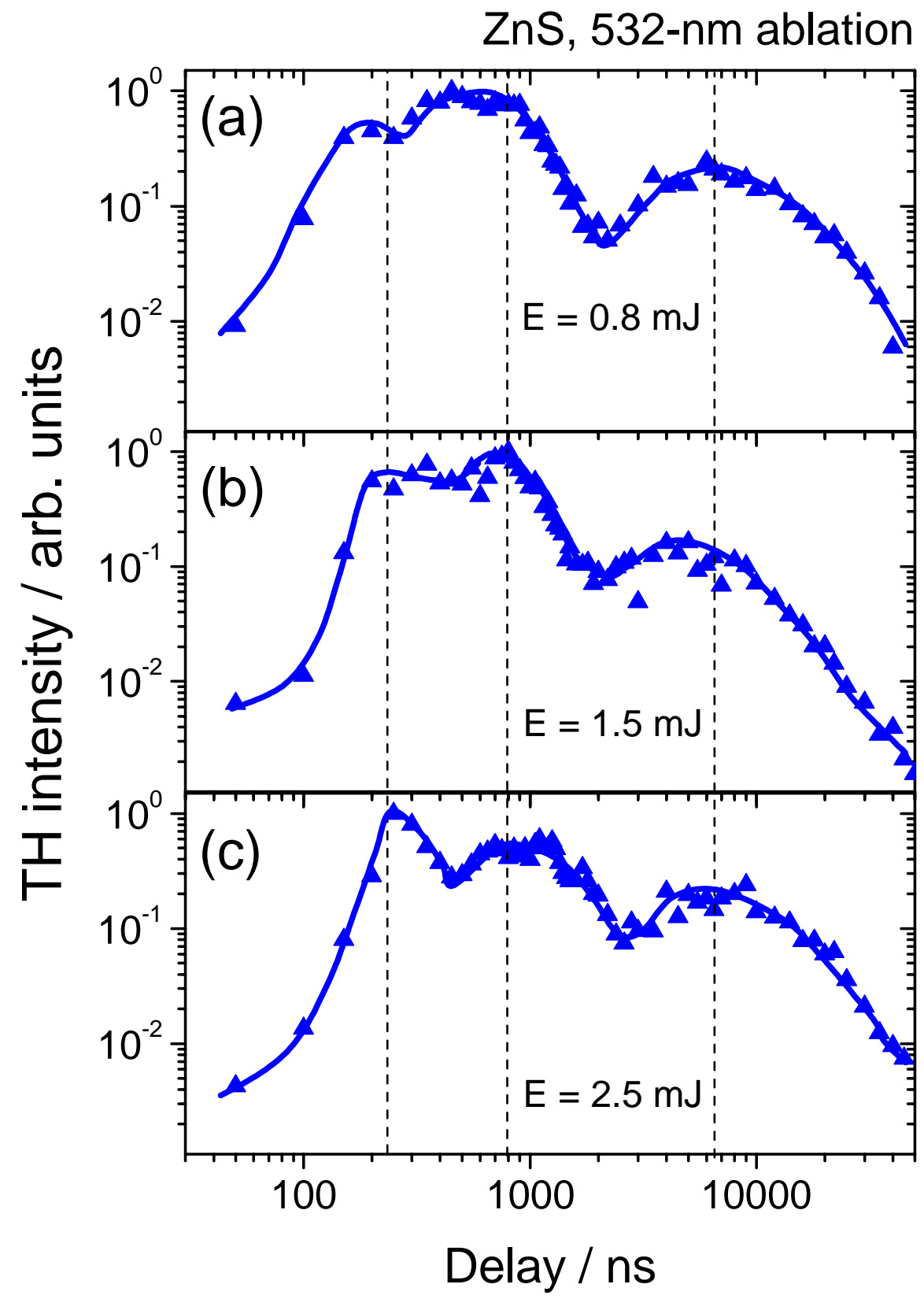

Figure 6 